\title{
SiGe Circuits for Spread Spectrum Automotive Radar
}

\author{
Saverio Trotta ${ }^{1,3}$, Bernhard Dehlink ${ }^{2,3}$, Herbert Knapp ${ }^{2}$, Klaus Aufinger ${ }^{2}$, Thomas F. Meister ${ }^{2}$, Josef Böck ${ }^{2}$, \\ Werner Simbürger ${ }^{2}$, and Arpad L. Scholtz ${ }^{3}$ \\ ${ }^{1}$ Now with Freescale Semiconductor GmbH, Schatzbogen 7, D-81829 Munich, Germany \\ ${ }^{2}$ Infineon Technologies AG, Am Campeon 1-12, D-81726 Munich, Germany \\ ${ }^{3}$ Vienna University of Technology, Karlsplatz 13, A-1040 Vienna, Austria \\ E-mail: Saverio.Trotta@freescale.com
}

\begin{abstract}
This paper presents circuits in SiGe bipolar technology for spread spectrum automotive radar applications in the band from 77 to $81 \mathrm{GHz}$. They cover the transmit and receive paths. The transmitter integrates a voltage controlled oscillator, a prescaler by 64, a 10 bit linear feedback shift register (LFSR), and a biphase modulator. The system has been optimized in order to achieve a range resolution less than $12 \mathrm{~cm}$ and an unambiguous range of $124 \mathrm{~m}$. The quadrature receiver frontend consists of a single-ended low-noise amplifier (LNA), balancedto-unbalanced converters, two fully differential direct-conversion mixers, LO buffer amplifiers, and a branchline coupler for $I / Q$ generation. The presented systems show that millimeter-wave circuits in SiGe technology can achieve high integration levels along with high performance. They are well suited for application in spread spectrum automotive radar systems.
\end{abstract}

\section{INTRODUCTION}

In a spread spectrum system the transmitted signal is spread over a frequency band, which is much wider than the minimum bandwidth required to transmit the information [1]. Automotive radar systems can take advantage of spread spectrum techniques because of their interference rejection, immunity from noise and multipath distortion, and high resolution ranging properties. In addition, there is no need for high-speed, fast-settling frequency synthesizers. Moreover, spread spectrum techniques can improve the reliability of automotive radars. The data from different sensors on different cars can be combined in order to observe the complete car environment. Thus a spread spectrum radar system will allow to share the same bandwidth also for datalink needed by car-to-car communication systems [2].

Pseudo noise (PN) signal processing along with directsequence modulation has been widely used for developing powerful spread spectrum ranging systems. These concepts are also well suited for short-range automotive radar applications in the $79 \mathrm{GHz}$ band. A simplified transmitter architecture is shown in Fig. 1. The spread spectrum signal is generated by means of direct sequence technique: the carrier is modulated with a pseudo noise sequence (code). Compared to other spread spectrum systems, pulse radar for example, the PN-radar allows to lower the maximum output power level of the transmit signal because it is continuously active. For shortrange applications (e.g. 1 to 30 meter), there is the need to use ultra-wideband (UWB) techniques since there is a direct

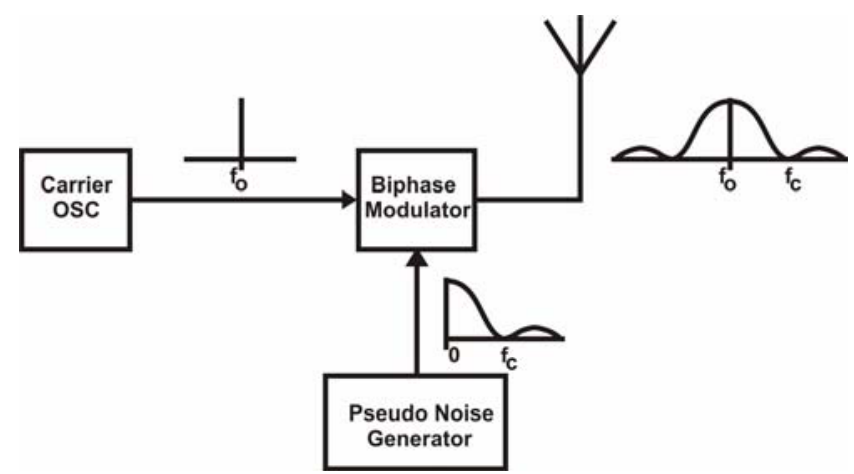

Figure 1. Simplified architecture of a spread spectrum transmitter.

relation between the bandwidth of the transmit signal $\left(\mathrm{f}_{\mathrm{C}}\right.$ in Fig. 1) and the achievable range resolution $d_{\min }[3,4]$ :

$$
d_{\text {min }} \leq c / 2 f_{C}
$$

where $\mathrm{c}$ is the speed of light. Another performance characteristic of a ranging system is the supported unambiguous range $d_{\max }$, which is the maximum distance that the system is able to detect:

$$
d_{\max } \leq c / 2 T_{P},
$$

where $T_{P}=N / f_{C}$ is the period of the pseudo random sequence and $N$ the number of bits. In order to keep the range resolution small and at the same time support large unambiguous range, the chip duration $T_{C}=1 / f_{C}$ is required to be small while $N$ has to be sufficiently large. For the system presented in this paper, $N=1023$ and $f_{C}=1.235 \mathrm{GHz}$ have been chosen in order to achieve a $d_{\max }$ of approximately $124 \mathrm{~m}$ and a $d_{\min }$ of less than $12 \mathrm{~cm}$. Moreover, in ranging systems based on spread spectrum techniques, a large value for $N$ is also needed to improve the peak of the autocorrelation function and lower the cross-correlation function. This allows the receiver to discriminate among spread spectrum signals generated by different sequences [10]. Considering a $79 \mathrm{GHz}$ carrier signal and a speed of $100 \mathrm{~km} / \mathrm{h}$, the Doppler signal is approximately $14.5 \mathrm{kHz}$. This is much lower than the PN-code repetition frequency of $1.2 \mathrm{MHz}$ (which represents the spacing between the discrete lines in the spectrum of the PN-signal) and therefore does not deteriorate the correlation response of the receiver in the PN-radar [5]. 


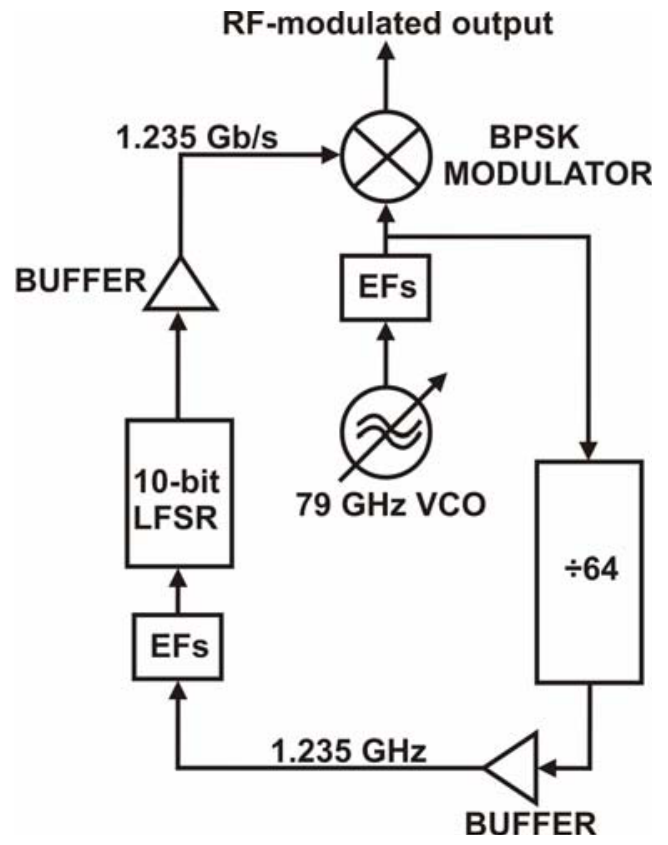

Figure 2. Block diagram of the transmitter.

Various radar systems require the use of in-phase and quadrature signals for the determination of the distance of the target and its relative velocity. In pulsed Doppler-radar systems, the received signal and the reference signal can be out-of-phase such that coherent detection results in zero output. No information about the target's relative velocity can therefore be extracted [6]. A second branch that is shifted in phase by $90^{\circ}$ yields the desired information in that case. The combination of both in-phase $\left(0^{\circ}, \mathrm{I}\right)$ and quadrature $\left(90^{\circ}, \mathrm{Q}\right)$ components using $\sqrt{\mathbf{S}_{\mathrm{I}}^{2}+\mathbf{S}_{\mathrm{Q}}^{2}}$ results in no loss of information. Spread-spectrum radar systems correlate the received signal with the reference PN-sequence to measure the distance to the target. The delayed reference and the incoming signal are incoherent, therefore the correlation can also yield zero output. For that case, the correlation with I and Q versions of the received signal is needed [7].

\section{CIRCUIT DESIGN}

\section{A. Transmitter}

The block diagram of the spread-spectrum transmitter chip is shown in Fig. 2. The system is fully differential and consists of four main blocks: a $79 \mathrm{GHz}$ VCO, a prescaler, a biphase modulator, and a pseudo-noise sequence generator. The clock signal which drives the $\mathrm{PN}$-source is generated on the same chip. The VCO is based on the chip presented in [8] and has a center frequency of $79 \mathrm{GHz}$. The differential signal generated by the VCO drives the biphase modulator and the prescaler.

The prescaler is used to downconvert the VCO signal to $1.235 \mathrm{GHz}$. It consists of six divider stages. The first stage is a dynamic frequency divider. Dynamic frequency dividers achieve higher operating frequencies than static frequency dividers at comparable or lower power dissipation. Therefore they are an attractive choice for the first stage of a frequency divider chain at the output of the 77-GHz VCO. In contrast to injection-locked dividers, which suffer from a narrow locking

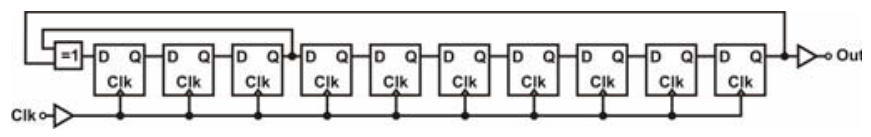

Figure 3. A 10 stages pseudo random bit sequence generator.

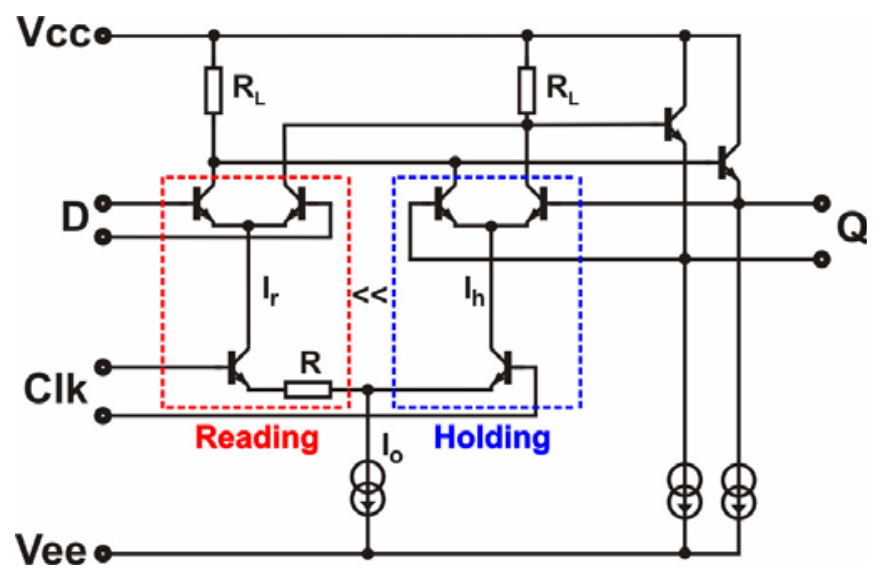

Figure 4. Latch schematic.
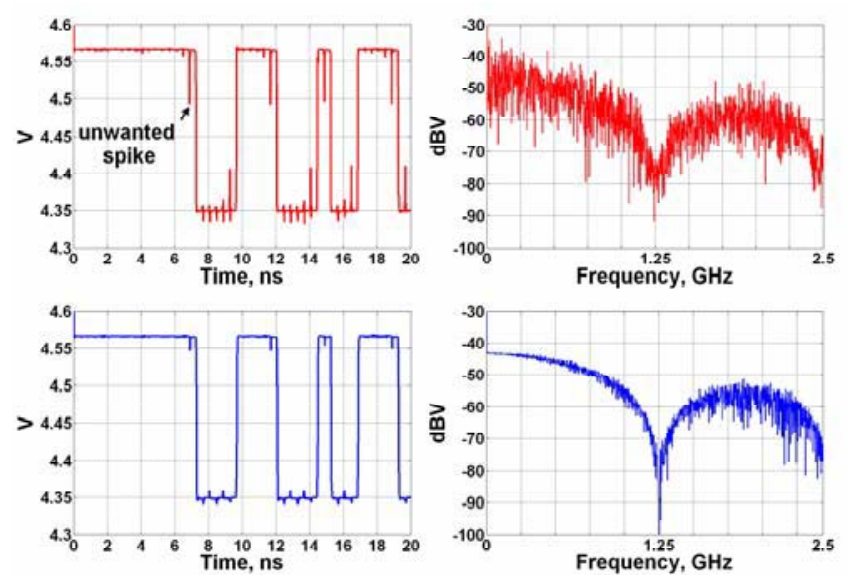

Figure 5. Simulation results of the PRBS: effect on the spectrum due to spikes in the pseudo noise signal. The quality of the output signal can be improved (lower figures) by using the resistor $\mathrm{R}$ in the latch.

range, regenerative dividers are able to operate over a frequency range of approximately $3: 1$. This provides sufficient margin to prevent a reduction of the usable VCO tuning range [9]. The other five stages are static dividers optimized for low power consumption. Since the signal from the prescaler has to drive all the clocked stages in the PN-generator, a buffer has been connected at the output of the last divider stage.

The PN-sequence is generated by a full-rate pseudo random bit sequence (PRBS) generator (Fig. 3). The chip rate of the PN-sequence is $1.235 \mathrm{~Gb} / \mathrm{s}$. Since $\approx 90 \%$ of the signal power of the PN-sequence is contained in the main lobe, the spread spectrum system will require a bandwidth of approximately $2.5 \mathrm{GHz}$. The PRBS generator consists of a clock input stage, a linear feedback shift register (LFSR) $[1,10]$, and an output buffer. Three cascaded emitter followers are used in the clock input stage in order to shift down the DC level of the clock signal and drive the LFSR. The shift register contains $n=10$ stages and generates a maximum-length 
sequence of $N=2^{n}-1=1023$ bits. The maximum-length sequence (M-sequences) are the longest codes generated by a given shift register. They have good autocorrelation property $[1,10]$. With a 10 stage LFSR it is possible to generate 10 different M-sequences [1]. The sequence of the PRBS is set by the output signals from the 10 stages which are fed back and applied to the first stage via an XOR. By using a multiplexer it is possible to select the feedback lines and thereby set the sequence. In this way, radars mounted on different cars can transmit different sequences avoiding potential interferences. In our first test chip, we did not implement the multiplexer. In this case the sequence is generated by the feedback signals from the third and the last stages (Fig. 3). Each stage in the PRBS is a delay element which has been implemented as a flipflop. The performance of the overall system at $79 \mathrm{GHz}$ strongly depends on the quality of the baseband PN-signal and it is quite challenging to integrate baseband together with high-frequency blocks. Recently, new techniques have been presented to improve the performance of flip-flops connected in a divider configuration $[11,12]$. Such techniques are based on asymmetric current levels in the reading and holding paths in each latch. This asymmetry leads to a longer reading time against a shorter holding time which makes the flip-flop faster. In order to improve the shape of the output signal from the PRBS, in our design the concept of asymmetry has been used, but in the opposite way. As shown in Fig. 4, in each latch a resistor has been placed in the reading path to unbalance the tail current $\mathrm{I}_{\mathrm{o}}$ in the holding path. In this way, the holding time will be longer than the reading time. Because of this asymmetry, spikes in the output signals are avoided during clock transitions, as shown in Fig. 5. In the third stage another differential pair has been also implemented to force the initial condition in the LFSR. In order to achieve a fast switching behavior at the upper differential pairs of the modulator, an output buffer has been integrated in the PRBS to achieve a maximum differential output swing of $1.4 \mathrm{~V}_{\mathrm{pp}}$.

The biphase modulator shifts the baseband spectrum of the PRBS to the carrier frequency. The fully differential biphase modulator is implemented as a Gilbert cell and shown in Fig. 6. The lower differential pair of the modulator is driven by the $79 \mathrm{GHz}$ carrier and the upper pairs by the PN-signal. Since the bit rate of the PN-signal is very low compared to the frequency of the carrier, the modulator works mostly as a cascode thereby improving the performance. Transmission lines are used as load in the modulator and also for on-chip matching.

\section{B. Quadrature receiver}

The quadrature receiver frontend consists of a single-ended low-noise amplifier (LNA), balanced-to-unbalanced converters to transform the single-ended signal to differential signals, two fully differential direct-conversion mixers, LO buffer amplifiers, and a branchline coupler [13]. An overview of the implemented circuit is depicted in Fig. 7.

The design of the LNA is similar to the one presented in [14]. It consists of three common-emitter (CE) stages, the first one is shown in Fig. 8. Stages two and three basically have the same topology. The input of the LNA is matched with transmission lines for a return loss larger than $10 \mathrm{~dB}$

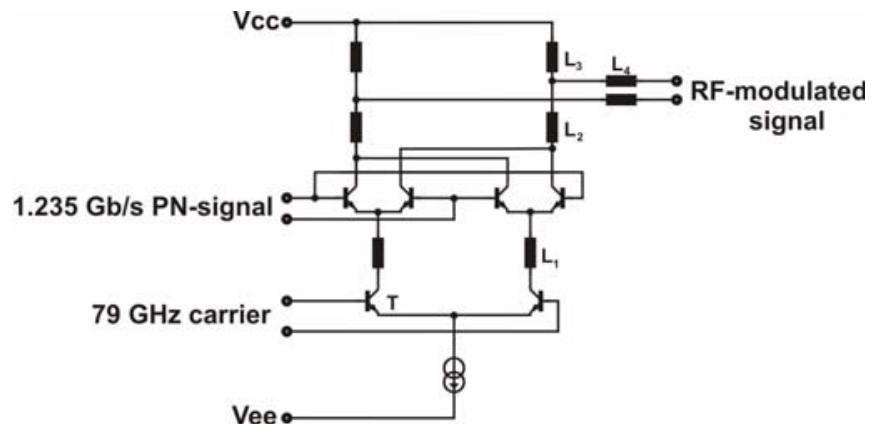

Figure 6. BPSK-modulator schematic. $\mathrm{L}_{1}, \mathrm{~L}_{2}, \mathrm{~L}_{3}$ and $\mathrm{L}_{4}$ are microstrip-line elements, all with inductive behavior.

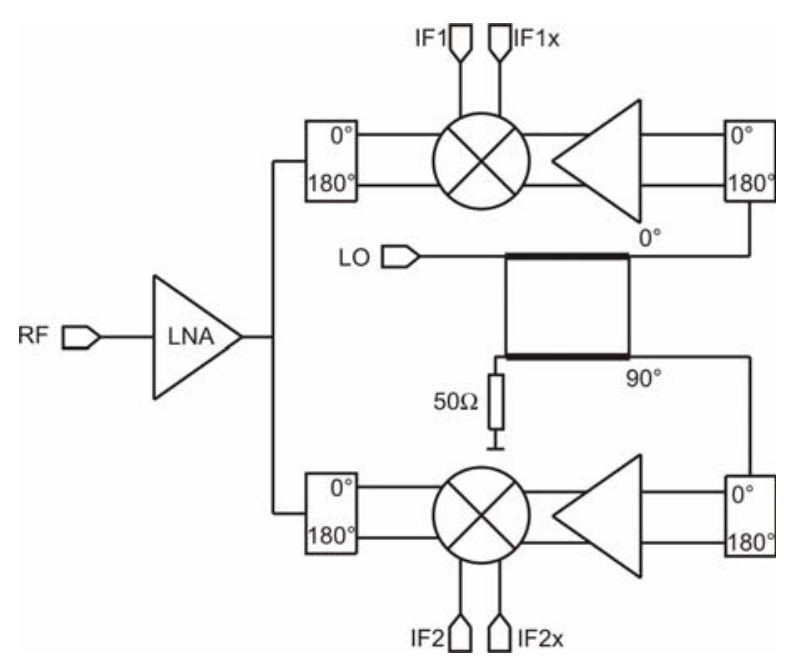

Figure 7. Block diagram of the IQ frontend.

(VSWR $<2: 1)$. The simulated noise figure of the LNA is $6.5 \mathrm{~dB}$ in the frequency range from $75 \mathrm{GHz}$ to $86 \mathrm{GHz}$. Interstage matching is accomplished by an LC matching network $\left(\mathrm{L}_{2}, \mathrm{C}_{3}\right)$. Here, the inductance is replaced by a transmission line that is shorted for high frequencies. The transformed input impedance of the following stage determines the load impedance (the gain) of the $\mathrm{CE}$ transistor $\mathrm{Q}_{1}$. The output of the LNA is matched with transmission lines to a $50 \Omega$ interface, taking into account the parasitic pad capacitance. The measured gain of a stand-alone version of the LNA is $12 \mathrm{~dB}$. The LNA consumes $32 \mathrm{~mA}$.

The power splitting of the RF signal is done as follows: Two transmission lines of $220 \mu \mathrm{m}$ length transform the input impedance of both RF baluns to a low impedance. This value is transformed to the $50 \Omega$ interface by a series transmission line and a shunt capacitor.

The RF and LO baluns are LC-baluns (Fig. 9) where the inductors are replaced by transmission lines [15]. With this type of balun, a single-ended to differential conversion and impedance matching is achieved simultaneously. The insertion loss of one balun is $1.6 \mathrm{~dB}$.

The direct-conversion mixers are based on the high-level mixer presented in [16]. The mixer is designed for high impedance external loads, so the internal load of $2 \times 400 \Omega$ 


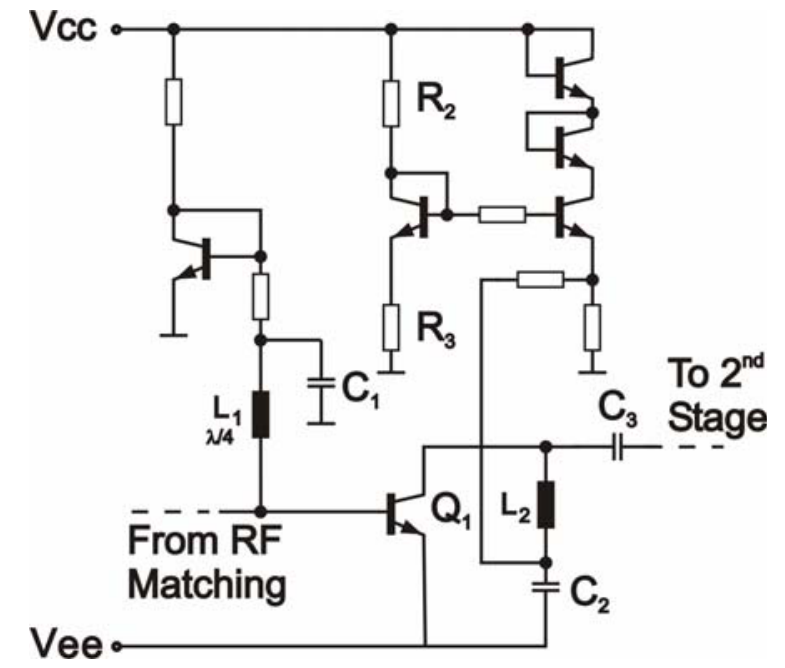

Figure 8. One stage of the low-noise amplifier. $\mathrm{L}_{1}$ and $\mathrm{L}_{2}$ are microstrip-line elements, all with inductive behavior.

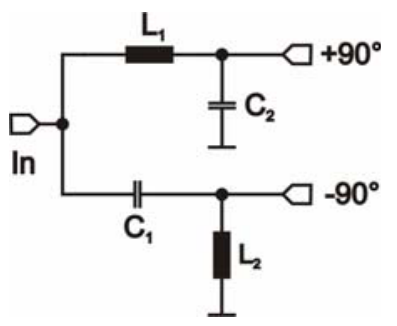

Figure 9. Schematic diagram of the balun. $\mathrm{L}_{1}$ and $\mathrm{L}_{2}$ are microstrip-line elements, all with inductive behavior.

and the bias current of $6 \mathrm{~mA}$ determine the conversion gain. The simulated noise figure of one mixer is $12 \mathrm{~dB}$, and the conversion gain is $24 \mathrm{~dB}$. The current consumption of one mixer, including biasing, is $11 \mathrm{~mA}$.

To maintain a high voltage swing at the LO port of the mixer, which is essential for good noise performance, the LO signal is applied to the four switching transistors via an LO buffer. The simulated gain of the buffer is $10 \mathrm{~dB}$ in the frequency range around $80 \mathrm{GHz}$. The buffers consume $60 \mathrm{~mA}$ each.

The $0^{\circ} / 90^{\circ}$ phase shift between the two IF output ports is achieved by the use of a branchline coupler at the LO port of the front-end. The isolated port of the coupler is terminated with $50 \Omega$ to broaden the coupler's bandwidth. Transmission lines match the input impedance of the coupler to $50 \Omega$, taking into account the parasitic pad capacitance. The four branches have a length of $480 \mu \mathrm{m}$ each, which equals $\lambda / 4$ at $80 \mathrm{GHz}$.

\section{TECHNOLOGY}

The chips are manufactured in an advanced $200 \mathrm{GHz} f_{T}$ $\mathrm{SiGe}: \mathrm{C}$ bipolar process based on the technology presented in [17]. The transistors achieve the highest $\mathrm{f}_{\mathrm{T}}$ at a current density of $5 \mathrm{~mA} / \mu \mathrm{m}^{2}$. Shallow and deep trench isolation are used. The transistors are fabricated with a double-polysilicon selfaligned emitter-base configuration with a SiGe:C base. This base is integrated by selective epitaxial growth. The transistors

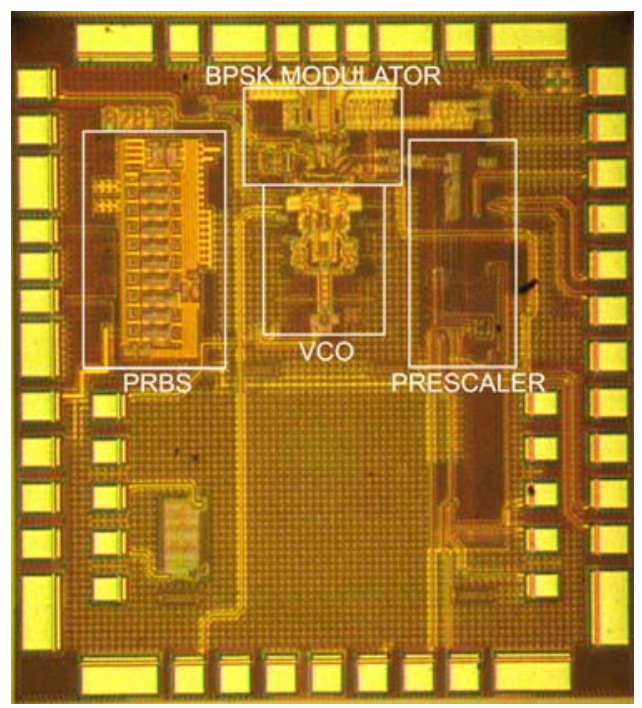

Figure 10. Chip photo of the spread spectrum transmitter $\left(1128 \times 1028 \mu \mathrm{m}^{2}\right)$.

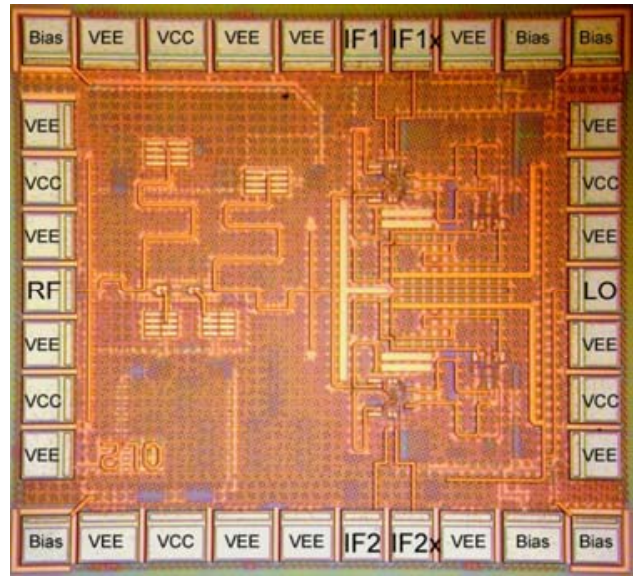

Figure 11. Chip photo of the quadrature receiver $\left(1100 \times 1000 \mu \mathrm{m}^{2}\right)$.

have a minimum emitter mask width of $0.35 \mu \mathrm{m}$, resulting in an effective emitter width of $0.18 \mu \mathrm{m}$. The technology additionally features high-voltage transistors with a $B V_{C E O}$ of $5.0 \mathrm{~V}$ [18]. Four metal layers, MIM-capacitors, varactors, and different types of resistors are also included. The chip photograph is depicted in Fig. 10 and 11. The size of the chip is $1128 \times 1028 \mu^{2}$ for the spread spectrum transmitter and $1100 \times 1000 \mu \mathrm{m}^{2}$ for the quadrature receiver.

\section{MEASUREMENT RESUlTS}

All measurements were performed on-wafer with probes. The temperature was kept at a constant level of $25^{\circ} \mathrm{C}$. All offchip losses from the measurement setup were de-embedded from the measurement results.

\section{A. Transmitter}

The complete spread spectrum transmitter draws $750 \mathrm{~mA}$ from a $+5.5 \mathrm{~V}$ supply. The baseband output from the PRBS was connected to a spectrum analyzer. The RF-modulated signal was connected via a W-band waveguide to a W-band down-conversion mixer, which down-converts the $79 \mathrm{GHz}$ 
modulated signal to an IF of $4.646 \mathrm{GHz}$. This output was applied to a second spectrum analyzer. The measurement results are shown in Figs. 12 and 13. The well-defined shape of the baseband signal validates the technique used in the design of the LFSR. Its quality also determines the precise shape of the up-converted signal, which shows only a small feedtrough of the carrier. The slight asymmetry in the measured spectrum shape of the up-converted signal is due to the variation of the conversion loss of the down-conversion mixer at different IF frequencies. The spectral nulls at $\Delta \mathrm{f}= \pm 1.235 \mathrm{GHz}$ can be clearly seen in the baseband and upconverted signal spectra. The code repetition frequency of the pseudo noise signal is shown in Fig. 14. The measured differential output power of the modulator is $+1.5 \mathrm{dBm}$, which is large enough for short-range applications without an additional power amplifier (PA) stage.

\section{B. Quadrature receiver}

The receiver draws $195 \mathrm{~mA}$ from a $+5.5 \mathrm{~V}$ supply. The LO power level was set to deliver $+1 \mathrm{dBm}$ to the chip. The mixers are designed for high impedance external loads, thus external voltage followers with an input impedance of $100 \mathrm{k} \Omega$ were attached to the output of the mixers. This provides matching to the $50 \Omega$ measurement environment. The differential IF signal was combined with a $180^{\circ}$ low-frequency hybrid. The noise figure was measured using HP's 8970B noise figure meter. The single-sideband (SSB) noise figure and the conversion gain are depicted in Fig. 15. At $79 \mathrm{GHz}$, the noise figure is $11 \mathrm{~dB}$ and the conversion gain $32 \mathrm{~dB}$. The $90^{\circ}$ phase difference between the in-phase and the quadrature output is a crucial parameter for the performance of precision sensor systems. Measurement results show that the relative phase deviation from $90^{\circ}$ is within $\pm 8^{\circ}$ from $75 \mathrm{GHz}$ to $88 \mathrm{GHz}$. Figure 16 shows the conversion gain and noise figure imbalance of the two mixer outputs. The gain differs by less than $0.7 \mathrm{~dB}$. The difference in noise figure is smaller than $0.4 \mathrm{~dB}$. The plot of the differential IF output voltage versus $\mathrm{RF}$ input power is shown in Fig. 17. Due to the external high impedance load of the mixer, the output voltage is depicted instead of the output power. The input-referred $1 \mathrm{~dB}$ compression point is $-19 \mathrm{dBm}$.

\section{CONCLUSION}

In this paper design considerations for SiGe circuits for spread spectrum automotive radar applications in the band from 77 to $81 \mathrm{GHz}$ have been reported. A complete transmitter at $79 \mathrm{GHz}$ with integrated baseband signal generation has been demonstrated. The chip provides $+1.5 \mathrm{dBm}$ differential output power, which is enough for short-range radar applications without an additional PA stage. The range resolution is less than $12 \mathrm{~cm}$. The main lobe of the spread spectrum RF signal occupies a bandwidth of $\approx 2.5 \mathrm{GHz}$. The quadrature receiver features a conversion gain of more than $28 \mathrm{~dB}$ and a noise figure of $11 \mathrm{~dB}$ over a frequency range from $75 \mathrm{GHz}$ to $86 \mathrm{GHz}$. The two IF outputs are within $8^{\circ}$ of $90^{\circ}$ from $75 \mathrm{GHz}$ to $88 \mathrm{GHz}$. The gain imbalance between these outputs is smaller than $0.7 \mathrm{~dB}$, and the imbalance in noise figure is smaller than $0.4 \mathrm{~dB}$. The input-referred $1 \mathrm{~dB}$ compression point is $-19 \mathrm{dBm}$. The performance of these circuits indicates their suitability for precision sensing applications at $79 \mathrm{GHz}$.

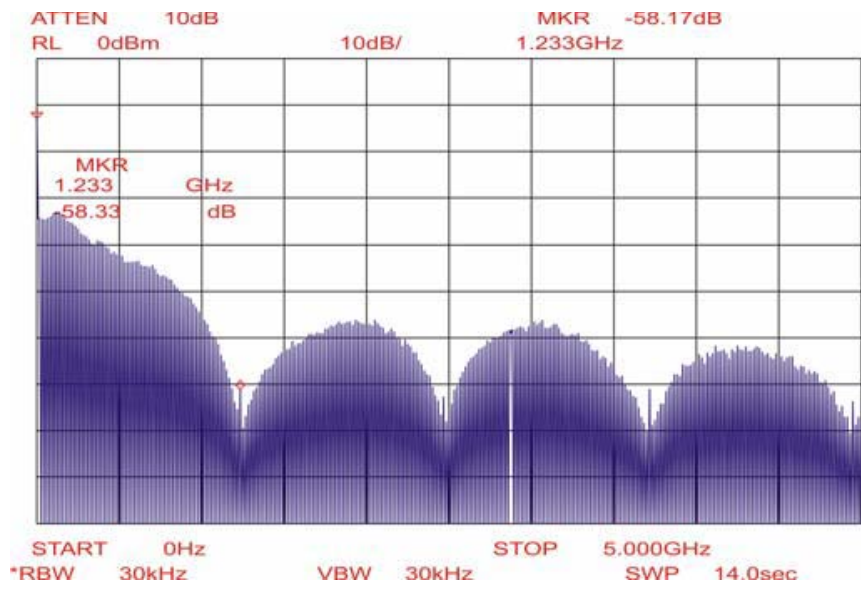

Figure 12. Measured pseudo noise baseband signal spectrum. Spectral nulls at $\Delta \mathrm{f}= \pm \mathrm{f}_{\mathrm{c}}=1.235 \mathrm{GHz}$.

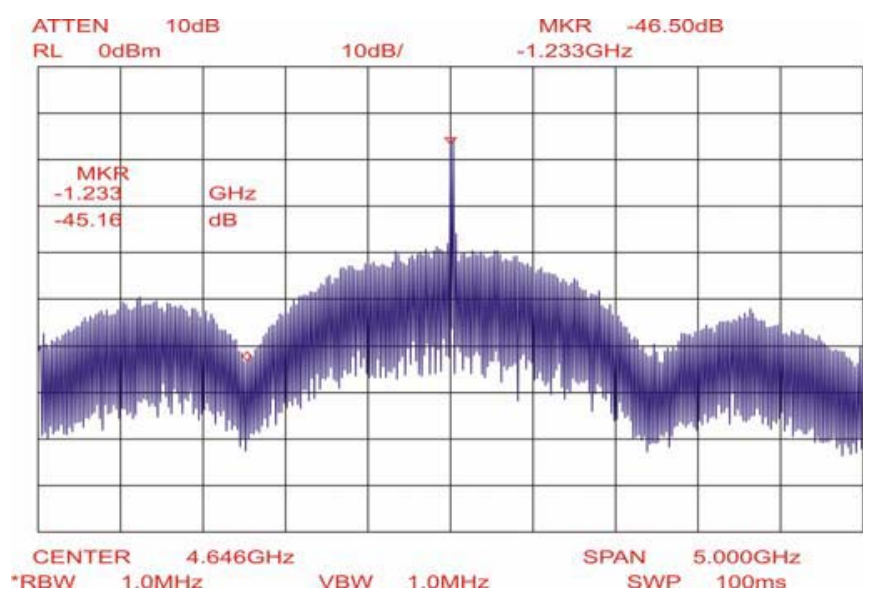

Figure 13. Measured RF-modulated signal spectrum. Free-running VCO; the $79 \mathrm{GHz}$ PN-signal was down-converted to an IF of $4.646 \mathrm{GHz}$. Spectral nulls at $\Delta \mathrm{f}= \pm \mathrm{f}_{\mathrm{c}}=1.235 \mathrm{GHz}$

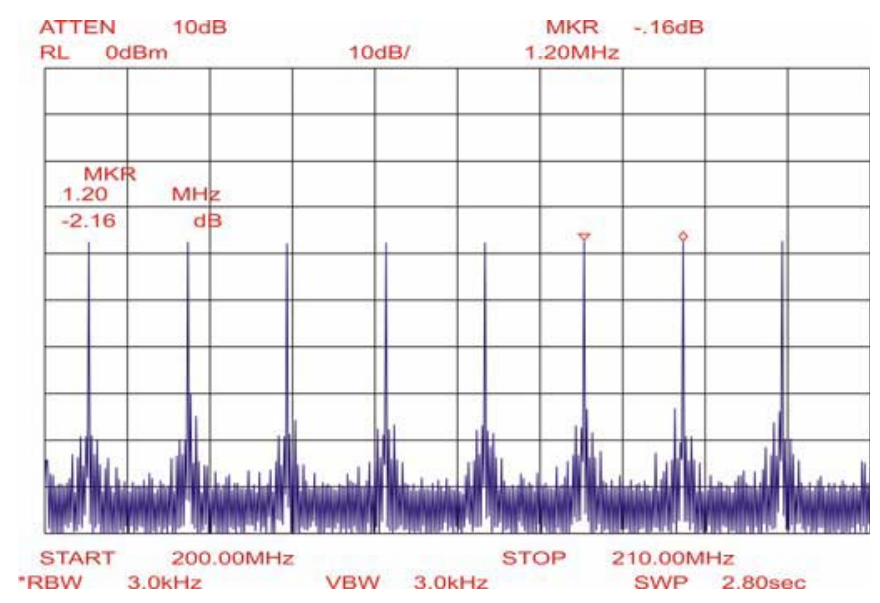

Figure 14. Measured discrete lines of the baseband signal spectrum. The PNcode repetition frequency is $f_{c} / N=1.2 \mathrm{MHz}$.

From the technology point of view, the obtained results show that very high integration levels in millimeter-wave frequency ranges are feasible in $\mathrm{SiGe}$ bipolar technology. 


\section{ACKNOWLEDGMENT}

This work was supported by the German Bundesministerium fuer Bildung und Forschung (BMBF) under contract $10 \mathrm{M} 3161 \mathrm{~A}$ (KOKON).

\section{REFERENCES}

[1] R.C. Dixon, Spread Spectrum Systems with Commercial Application, John Wiley \& Sons, $3^{\text {rd }}$ Edition, 1994.

[2] V. Wider, J. Detlefsen, U. Siart, J. Büchlert, and M. Wagner, "Automotive Radar Sensor with Communication Capability," IEEE ECWT Digest, pp. 305-308, Oct. 2004.

[3] H. Veenstra, E. van der Heijden, and D. van Goor, " $15-27 \mathrm{GHz}$ Pseudo-Noise UWB transmitter for short-range automotive radar in a production SiGe technology," IEEE Proceedings of ESSCIRC, pp. 275-278, Sept. 2005.

[4] S. Trotta, H. Knapp, D. Dibra, K. Aufinger, T. F. Meister, J. Böck, W. Simbürger, and A. L. Scholtz, "A 79GHz SiGeBipolar Spread Spectrum TX for Automotive Radar," IEEE ISSCC Digest, pp. 430-431, Feb. 2007.

[5] J. Detlefsen, E. Schmidhammer, and T. Troll, "Spread Spectrum Ranging for an ACC-Radar," IEEE Proceeding of ISSSTA, vol. 3, pp. 994-998, Sep. 1998.

[6] Royal School of Artillery, Principles of Pulsed Doppler Radar. Basic Science \& Technology Section (BST), pp. H03-1 - H0316, May 2004.

[7] V. Filimon, and J. Buechler, "A Pre-Crash Radar Sensor System Based on Pseudo-Noise Coding," IEEE MTT-s Digest, pp. 1415-1418, June 2000.

[8] H. Li and H.-M. Rein, "Millimeter-Wave VCOs With Wide Tuning Range and Low Phase Noise, Fully Integrated in a SiGe Bipolar Technology," IEEE J. Solid-State Circuits, vol. 38, pp. 184-191, Feb. 2003.

[9] H. Knapp, B. Dehlink, H.-P. Forstner, E. Kolmhofer, K. Aufinger, J. Böck, and T. F. Meister, "SiGe Circuits for Automotive Radar," IEEE SiRF Digest, pp. 231-236, Jan. 2007.

[10] H.-J. Zepernick and A. Finger, Pseudo Random Signal Processing -Theory and Application, John Wiley \& Sons, 2005.

[11] Y. Suzuki, Z. Yamazaki, Y. Amamiya, S. Wada, H. Uchida, C. Kurioka, S. Tanaka, and H. Hida, "120-Gb/s Multiplexing and 110-Gb/s Demultiplexing ICs," IEEE J. Solid-State Circuits, vol. 39, pp. 2397-2402, Dec. 2004.

[12] S. Trotta, H. Knapp, T. F. Meister, K. Aufinger, J. Böck, W. Simbürger, and A. L. Scholtz, "110-GHz Static Frequency Divider in SiGe Bipolar Technology," IEEE CSICS Digest, pp. 291-294, Nov. 2005.

[13] B. Dehlink, H.-D. Wohlmuth, K. Aufinger, F. Weiss, and A. L. Scholtz, "An $80 \mathrm{GHz}$ SiGe Quadrature Receiver Frontend," IEEE CSICS Digest, pp. 197-200, Nov. 2006.

[14] B. Dehlink, H.-D. Wohlmuth, K. Aufinger, T. F. Meister, J. Böck, and A. L. Scholtz, "A low-noise amplifier at $77 \mathrm{GHz}$ in SiGe:C bipolar technology," IEEE CSICS Digest, pp. 287-290, Nov. 2005.

[15] W. Bakalski, W. Simbürger, H. Knapp, H.-D. Wohlmuth, and A. L. Scholtz, "Lumped and distributed lattice-type LC-baluns," IEEE MTT-s Digest, pp. 209-212, June 2002.

[16] H.-D. Wohlmuth and W. Simbürger, "A high IP3 RF Receiver Chip Set for Mobile Radio Base Stations up to $2 \mathrm{GHz}$," IEEE J. Solid-State Circuits, vol. 36, no. 7, pp. 1132-1137, July 2001.

[17] J. Böck, H. Schäfer, K. Aufinger, R. Stengl, S. Boguth, R. Schreiter, M. Rest, H Knapp, M. Wurzer, W. Perndl, T. Böttner, and T.F. Meister, "SiGe bipolar technology for automotive radar applications", IEEE BCTM Digest, pp. 84-87, Sept. 2004.

[18] R. K. Vytla, T. F. Meister, K. Aufinger, D. Lukashevich, S. Boguth, H. Knapp, J. Böck, H. Schäfer, and R. Lachner, "Simultaneous Integration of SiGe High Speed Transistors and
High Voltage Transistors", IEEE BCTM Digest, pp. 61-64, Oct. 2006.

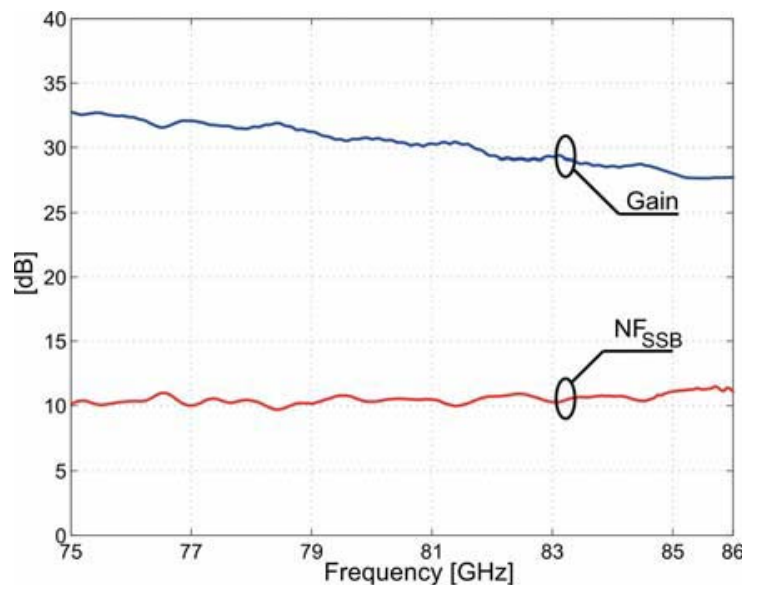

Figure 15. Measured single-sideband noise figure and conversion gain of the quadrature receiver $(\mathrm{IF}=10 \mathrm{MHz})$.

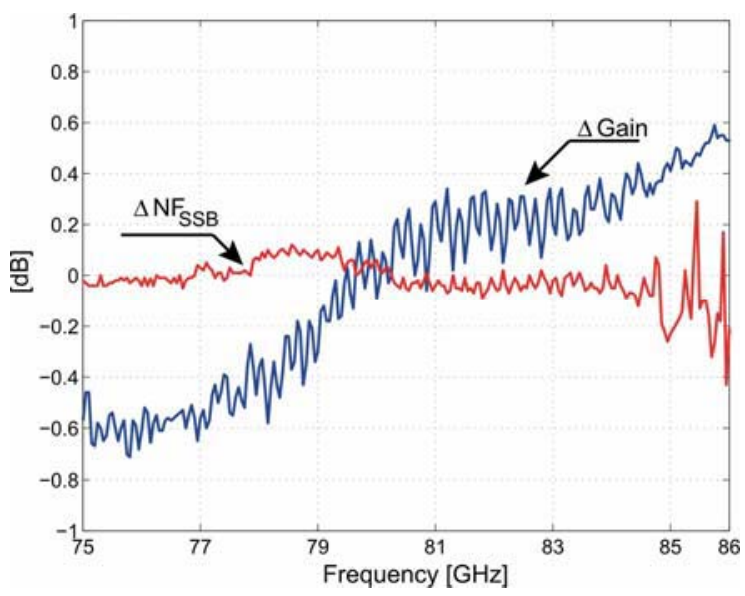

Figure 16. Measured gain and noise figure imbalance of the two output ports $(\mathrm{IF}=10 \mathrm{MHz})$.

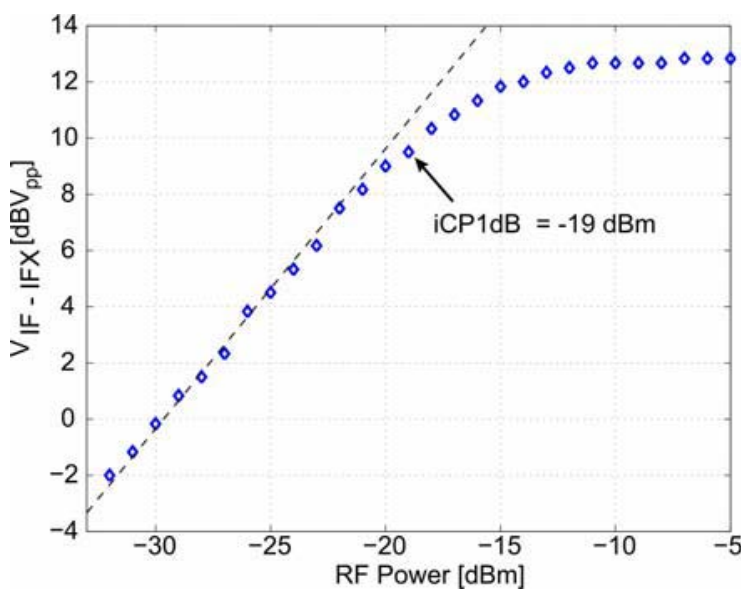

Figure 17. Measured input-referred 1-dB compression point of the quadrature receiver $(\mathrm{IF}=10 \mathrm{MHz})$. 